\title{
QUALIDADE DE VIDA DE PESSOAS COM DIABETES MELLITUS NA ATENÇÃO PRIMÁRIA: UMA REVISÃO INTEGRATIVA
}

\section{QUALITY OF LIFE OF PEOPLE WITH DIABETES MELLITUS IN PRIMARY CARE: AN INTEGRATIVE REVIEW}

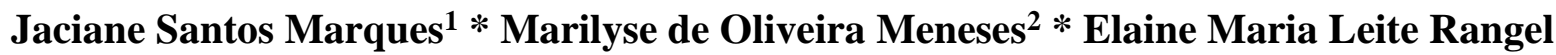 Andrade $^{3} *$ José Ramón Martínez-Riera $^{4} *$ Fernando Lopes e Silva Júnior ${ }^{*}$ Herla Maria Furtado Jorge $^{6} *$ Ana Roberta Vilarouca da Silva ${ }^{7}$}

\section{RESUMO}

Objetivo: analisar as evidências científicas disponíveis na literatura sobre a qualidade de vida de pessoas com Diabetes Mellitus na Atenção Primária. Método: a pesquisa foi realizada de março a abril de 2020 na MEDLINE e LILACS. Resultados: Analisou- se 16 estudos dos quais, dez foram publicados em inglês e seis em português, entre os anos de 2015 e 2020, todos com design observacional. Os países de origem dos estudos foram o Brasil com seis publicações, seguido da Malásia, Estados Unidos da América e Holanda com duas publicações e Kuwait, Alemanha, China e Índia com apenas uma. Após a análise, percebeu-se que a qualidade de vida das pessoas com diabetes está relacionada principalmente ao perfil sociodemográfico e clínico, com destaque para os fatores que influenciam de forma positiva ou negativa na qualidade de vida desta população. Conclusões: constatou-se que as pessoas com Diabetes Mellitus na Atenção Primária apresentam um bom padrão geral de qualidade de vida.

Palavras-chave: Diabetes Mellitus; Qualidade de Vida; Atenção Primária à Saúde.

\section{ABSTRACT}

Objective: to analyze the scientific evidence available in the literature on the quality of life of people with Diabetes Mellitus in Primary Care. Methodology: the survey was conducted from March to April 2020 on MEDLINE and LILACS. The search resulted in 16 studies for analysis, of which, ten were published in English and six in Portuguese, between the years 2015 and 2020, all with observational design. Results: the countries of origin of the studies were Brazil with six publications, followed by Malaysia, the United States of America and Holland with two publications and Kuwait, Germany, China and India with only one. After the analysis, it was noticed that the quality of life of people with diabetes is mainly related to the sociodemographic and clinical profile, with emphasis on the factors that have a positive or negative influence on the quality of life of this population. Conclusion: it was found that people with Diabetes Mellitus in Primary Care have a good general standard of quality of life

Keywords: Diabetes Mellitus; Quality of Life; Primary Health Care.

\footnotetext{
${ }^{1}$ Universidade Federal do Piauí, Teresina, Brasil, https://orcid.org/0000-0002-3452-5759

${ }^{2}$ Universidade Federal do Piauí, Teresina, Brasil, https://orcid.org/0000-0002-6883-3856

${ }^{3}$ Universidade Federal do Piauí, Teresina, Brasil, https://orcid.org/0000-0002-1772-7439

${ }^{4}$ Universidade de Alicante, Alicante Espanha, https://orcid.org/0000-0002-4926-6622

${ }^{5}$ Universidade Federal do Piauí, Teresina, Brasil, https://orcid.org/0000-0002-0273-6738

${ }^{6}$ Universidade Federal do Piauí, Teresina, Brasil, https://orcid.org/0000-0001-9706-5369

${ }^{7}$ Universidade Federal do Piauí, Teresina, Brasil, https://orcid.org/0000-0001-5087-4310 


\section{INTRODUÇÃO}

O Diabetes Mellitus (DM) é uma síndrome de etiologia múltipla, decorrente da falta de insulina e/ou incapacidade da mesma em desempenhar de forma adequada os seus efeitos ${ }^{(1)}$ sendo considerada um problema de saúde pública, de proporções epidêmicas, com elevada prevalência em todo o mundo ${ }^{(2)}$.

No Brasil, aproximadamente 16,8 milhões de indivíduos com idade entre 20 e 79 anos convivem com a doença, o equivalente a $11,4 \%$ da população nessa faixa etária. Quanto à mortalidade, cerca de 135.200 mortes são causadas por complicações do diabetes, o que coloca o país na quinta posição de causa mundial de mortes (3).

Esta condição aumenta de forma considerável os índices de morbimortalidade e representa uma das principais causas de mortalidade, insuficiência renal, amputação de membros inferiores, cegueira e doença cardiovascular ${ }^{(4)}$. O tratamento é realizado por meio de medidas medicamentosas e não medicamentosas com o intuito de controlar os níveis glicêmicos a fim de evitar complicações agudas e crônicas, promover a qualidade de vida (QV) e reduzir a mortalidade ${ }^{(5)}$.

A QV é definida como a percepção do indivíduo de sua posição na vida, no contexto da cultura e no sistema de valores nos quais ele vive e em relação aos seus objetivos, expectativas, padrões e preocupações (6). para a formulação e implementação de estratégias de cuidado para as pessoas com DM, a partir da identificação dos fatores que interferem na $\mathrm{QV}$, para que as intervenções sejam efetivas, específicas e capazes de minimizar ou prevenir o seu comprometimento ${ }^{(7)}$.

Nesse ínterim, a Atenção Primária à Saúde (APS), enquanto porta de entrada preferencial do Sistema Único de Saúde e promotora de ações voltadas à promoção, prevenção, diagnóstico, tratamento e reabilitação ${ }^{(8)}$, constitui-se em importante instrumento para enfrentamento das iniquidades e condições de vida e saúde da sociedade $^{(9)}$, o que viabiliza um cuidado integral, de forma que as intervenções sobre os fatores condicionantes e determinantes em saúde possam ser vivenciadas pelos usuários, família e comunidade de forma longitudinal.

$\mathrm{Na}$ literatura é reconhecido através de resultados de pesquisas um elevado número de fatores influenciáveis na QV dessas pessoas, tais como o uso de insulina, a idade, o sexo, a renda, a escolaridade, as complicações da doença, os fatores psicológicos, o conhecimento sobre a doença, as atitudes de cuidado com a sua saúde, entre outros $^{(2)}$.

Diante deste cenário, este estudo objetivou analisar as evidências científicas disponíveis na literatura sobre a qualidade de vida de pessoas com Diabetes Mellitus na Atenção Primária à Saúde. 


\section{MÉTODOS}

Trata- se de uma revisão integrativa da literatura caracterizada como um método que permite a inclusão de estudos de diferentes abordagens metodológicas, que possibilita a síntese e análise do conhecimento produzido, além de proporcionar intervenções mais eficazes e com melhor relação custo-benefício (10).

A revisão seguiu as seguintes etapas: seleção da questão norteadora; amostragem ou pesquisa bibliográfica; seleção, por pares, das pesquisas que compuseram a amostra; extração de dados dos estudos incluídos; avaliação e interpretação dos resultados; e apresentação da revisão ou síntese do conhecimento produzido ${ }^{(11)}$. A revisão foi conduzida de acordo com as recomendações de Itens de Relatório Preferenciais para Revisões Sistemáticas e Meta-análises (PRISMA-P).

Para a elaboração da questão norteadora, utilizou-se a estratégia PICo, na qual se definiu a população "pessoas com Diabetes Mellitus", fenômeno de interesse

Quadro 1- Descritores controlados e não controlados utilizados para construção da estratégia de busca nas bases MEDLINE E LILACS. Teresina, PI, Brasil, 2020.

\begin{tabular}{|c|c|l|}
\hline \multicolumn{2}{|c|}{} \\
\hline \multirow{2}{*}{ P } & DC & Diabetes Mellitus \\
\cline { 2 - 3 } & DNC & Diabete; Diabete Melito; Diabetes; Diabetes Melito \\
\hline \multirow{2}{*}{ I } & DC & Qualidade de Vida \\
\cline { 2 - 3 } & DNC & Qualidade de Vida Relacionada à Saúde \\
\hline Co & DC & Atenção Primária à Saúde \\
\hline
\end{tabular}




\begin{tabular}{|c|l|l|}
\hline \multicolumn{1}{|c|}{} & $\begin{array}{l}\text { Atendimento Básico; Atendimento Primário; Atendimento Primário de } \\
\text { Saúde; Atenção Básica; Atenção Básica de Saúde; Atenção Básica à } \\
\text { Saúde; Atenção Primária; Atenção Primária de Saúde; Atenção Primária } \\
\text { em Saúde; Cuidados Primários; Cuidados Primários de Saúde; Cuidados } \\
\text { Primários à Saúde; Cuidados de Saúde Primários; Primeiro Nível de } \\
\text { Assistência; Primeiro Nível de Atendimento; Primeiro Nível de Atenção; } \\
\text { Primeiro Nível de Atenção à Saúde; Primeiro Nível de Cuidado; Primeiro } \\
\text { Nível de Cuidados }\end{array}$ \\
\hline \multicolumn{1}{|c|}{} \\
\end{tabular}

Legenda: DC - Descritor Controlado; DNC - Descritor Não Controlado.

Fonte: elaborada pelos autores

Para a seleção dos estudos, foram estabelecidos os seguintes critérios de inclusão: estudos primários em português, inglês e espanhol, publicados nos últimos cinco anos, com texto completo disponível e que abordasse a temática do estudo. Foram excluídos estudos duplicados, teses e dissertações e revisões de literatura.

Para resumir os resultados, os pesquisadores preencheram um instrumento próprio composto pelas seguintes informações: autor principal, ano e país de publicação, amostra de participantes, objetivo, desenho do estudo, instrumentos utilizados para avaliar a QV e principais resultados e conclusões.

A análise e síntese dos dados foram realizadas de forma descritiva e as produções selecionadas foram organizadas em planilhas no programa Microsoft Excel, procedendo-se 
à construção de tabelas de acordo com as variáveis identificadas. Além disso, o material foi ordenado e classificado por semelhança semântica, o que possibilitou o desenvolvimento da discussão.

Nesta busca identificou- se inicialmente um total de 458 artigos científicos, dos quais 129 atenderam aos critérios de inclusão e foram selecionadas para este estudo. Após a leitura dos títulos e resumos, realizou-se a exclusão de 101 publicações por duplicidade nas bases de dados e por compreenderem estudos secundários, do tipo revisão da literatura. Desse modo, 16 estudos foram selecionados para compor a amostra da revisão, conforme mostra a figura 1 .

Figura 1 - Fluxograma representativo do processo de identificação, rastreamento, elegibilidade e inclusão dos artigos incluídos na revisão integrativa da literatura, Teresina, PI, Brasil, 2020.

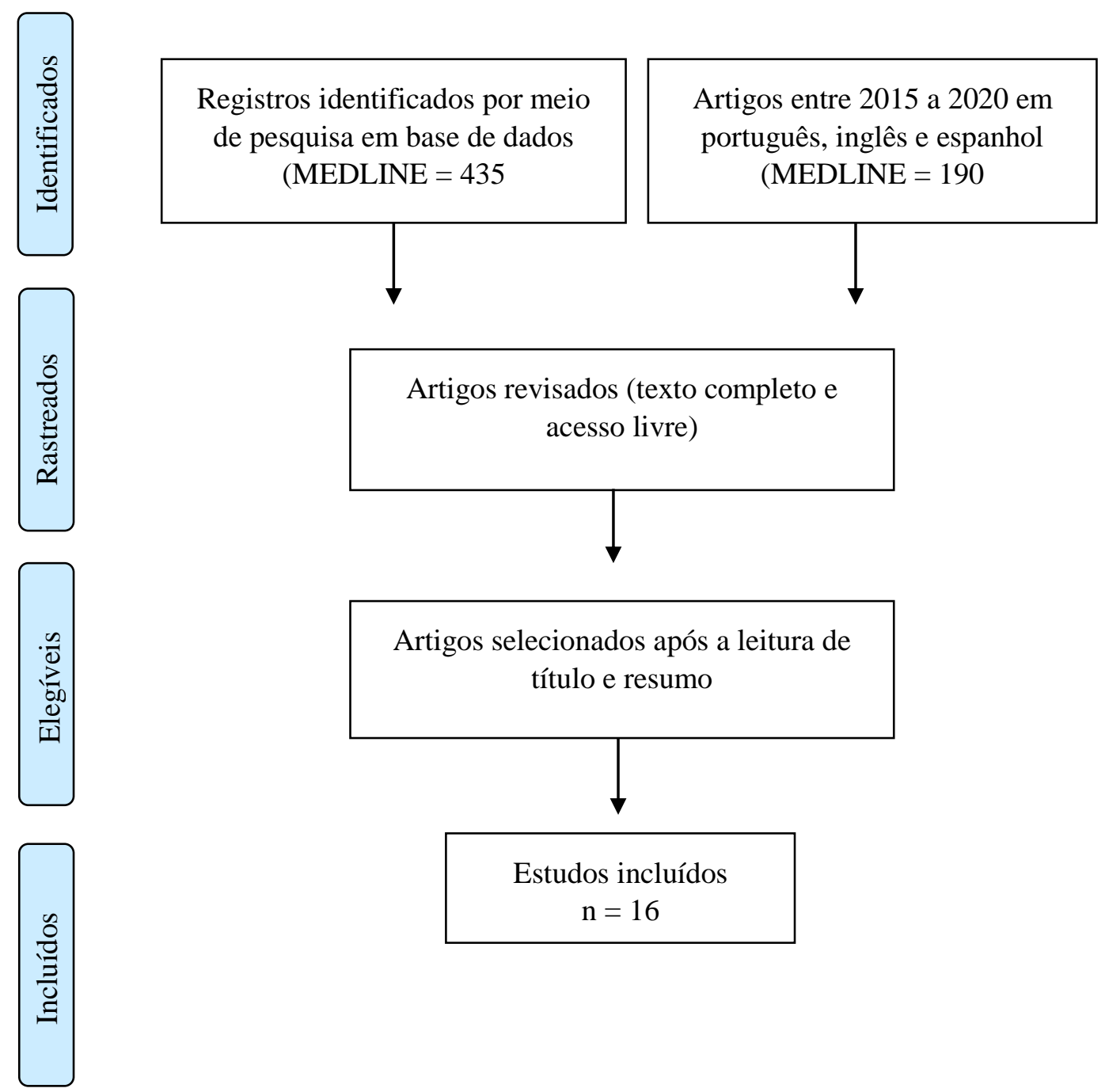

Fonte: dados da pesquisa 


\section{RESULTADOS}

Ao considerar a totalidade dos artigos selecionados, observa- se que com relação ao ano de publicação, os anos 2019, 2016 e 2015, destacaram-se com a maior quantidade de estudos publicados, ou seja, cada um com quatro, seguido dos anos 2017 e 2018 com duas publicações cada.

Quanto à localização geográfica onde os estudos foram realizados, seis eram do Brasil, seguido pela Malásia, Estados Unidos da América, e Holanda com duas publicações e Kuwait, Alemanha, China e Índia com apenas uma. Isso se reflete nos idiomas dos artigos apresentados, em que dez encontravam - se em inglês e seis artigos em português.

Quanto ao desenho metodológico, os 16 estudos analisados foram de natureza observacional. O tamanho das amostras envolvidas nos estudos variou de 50 a 1676 participantes. Nove questionários diferentes de avaliação de QV foram incluídos nesta revisão. O mais utilizado foi o Short Form- 12 Health Survey 2 (SF-12) em cinco estudos ${ }^{(13-}$ 17), quatro utilizaram o World Health Organization Quality of Life- Bref (WhoqolBref) $)^{(2,18-20)}$, três utilizaram o Medical Outcomes Study 36 - Item Short Form Health Survey $(\text { SF-36) })^{(21-23)}$, seguido do World Health Organization Quality of Life Assessment for Older Adults (WhoqolOld $)^{(2,20)}$ e do European Quality of Life 5 Dimensions (EQ-5D) $)^{(23,24)}$, com dois estudos e Problems Areas in Diabetes Scale $(B P A I D)^{(25)}$, Short Form- 6 Dimension (SF6D) ${ }^{(13)}$, Diabetes Quality of Life Measure (DQOL-Brasil) $^{(26)}$, Asian Diabetes Quality of Life (AsianDQOL) ${ }^{(27)}$, utilizado em apenas um estudo, conforme quadro 2 .

Quadro 2 - Caracterização dos artigos publicados entre 2015 e 2019. Teresina, PI, Brasil, 2020.

\begin{tabular}{|c|c|c|c|c|c|}
\hline $\mathbf{N}^{\mathbf{o}}$ & $\begin{array}{l}\text { Autor } \\
\text { principal, } \\
\text { país e } \\
\text { ano }\end{array}$ & $\mathbf{N}$ & Objetivo & $\begin{array}{l}\text { Tipo de } \\
\text { estudo }\end{array}$ & Instrumento \\
\hline 1 & $\begin{array}{l}\text { Leite ES } \\
\text { Brasil } \\
2015^{(25)}\end{array}$ & 68 & $\begin{array}{l}\text { Avaliar a qualidade de vida de idosos } \\
\text { com Diabetes Mellitus tipo } 2 \\
\text { acompanhados pelos profissionais da } \\
\text { atenção básica. }\end{array}$ & Observacional & BPAID \\
\hline 2 & $\begin{array}{l}\text { Chew BH } \\
\text { Malásia } \\
2015^{(18)}\end{array}$ & 650 & $\begin{array}{l}\text { Examinar a associação entre as variáveis } \\
\text { sociodemográficas, clínicas e a Qualidade } \\
\text { de vida relacionada a saúde (QVRS), bem } \\
\text { como cada um dos escores do domínio } \\
\text { WHOQOL-BREF, utilizando análises de } \\
\text { regressão multivariáveis. }\end{array}$ & Observacional & $\begin{array}{c}\text { WHOQOL- } \\
\text { BREF }\end{array}$ \\
\hline
\end{tabular}




\begin{tabular}{|c|c|c|c|c|c|}
\hline 3 & $\begin{array}{c}\text { Wong } \\
\text { CKH } \\
\text { China } \\
2015^{(13)}\end{array}$ & 298 & $\begin{array}{c}\text { Avaliar se o Programa de } \\
\text { Empoderamento do Paciente (PEP) } \\
\text { melhorou a QVRS em pacientes com } \\
\text { DM2, se o aumento do número de sessões } \\
\text { atendidos levaram a uma melhor QVRS e } \\
\text { quais componentes (genérico versus } \\
\text { componente específico do diabetes) as } \\
\text { intervenções foram associadas a uma } \\
\text { melhor QVRS. }\end{array}$ & Observacional & $\begin{array}{c}\text { SF-12 e SF- } \\
6 \mathrm{D}\end{array}$ \\
\hline 4 & $\begin{array}{l}\text { Dawson } \\
\text { AZ } \\
\text { Estados } \\
\text { Unidos } \\
2015^{(14)}\end{array}$ & 602 & $\begin{array}{l}\text { Investigar a saúde física sob a forma de } \\
\text { medidas biológicas, o componente } \\
\text { mental da qualidade de vida e } \\
\text { comportamentos de saúde conhecidos por } \\
\text { melhorar os resultados do diabetes. }\end{array}$ & Observacional & SF-12 \\
\hline 5 & $\begin{array}{l}\text { Adriaanse } \\
\text { MC } \\
\text { Holanda } \\
2016^{(15)}\end{array}$ & 1676 & $\begin{array}{l}\text { Estudar a prevalência, o impacto e a } \\
\text { relação dose-resposta de comorbidades } \\
\text { crônicas, condições de qualidade de vida } \\
\text { física e mental nos pacientes com } \\
\text { diabetes tipo } 2 \text {. }\end{array}$ & Observacional & SF-12 \\
\hline 6 & $\begin{array}{l}\text { Sousa EL } \\
\text { Brasil } \\
2016^{(21)}\end{array}$ & 68 & $\begin{array}{c}\text { Avaliar a qualidade de vida de idosos } \\
\text { acometidos por Diabetes Mellitus tipo } 2 \mathrm{e} \\
\text { identificar os fatores associados. }\end{array}$ & Observacional & SF-36 \\
\hline 7 & $\begin{array}{l}\text { Sreedevi } \\
\text { A } \\
\text { Índia } \\
2016^{(19)}\end{array}$ & 200 & $\begin{array}{c}\text { Determinar a confiabilidade, } \\
\text { construir validade discriminante do } \\
\text { WHOQOL-BREF traduzido entre pessoas } \\
\text { com diabetes tipo } 2 \text { e avaliar os } \\
\text { determinantes da QV. }\end{array}$ & Observacional & $\begin{array}{l}\text { WHOQOL- } \\
\text { BREF }\end{array}$ \\
\hline 8 & $\begin{array}{l}\text { Achuko O } \\
\text { Estados } \\
\text { Unidos } \\
2016^{(16)}\end{array}$ & 615 & $\begin{array}{c}\text { Examinar o caminho pelo qual a } \\
\text { discriminação influencia a qualidade de } \\
\text { vida de pacientes com diabetes tipo } 2 \text {. }\end{array}$ & Observacional & SF-12 \\
\hline 9 & $\begin{array}{l}\text { Corrêa K } \\
\text { Brasil } \\
2017^{(26)}\end{array}$ & 478 & $\begin{array}{l}\text { Este estudo objetivou avaliar a } \\
\text { associação entre qualidade de vida e } \\
\text { variáveis clínicas e sociodemográficas em } \\
\text { pacientes diabéticos tipo 2, após } \\
\text { iniciarem tratamento na Atenção Primária } \\
\text { e Especializada. }\end{array}$ & Observacional & DQOL-Brasil \\
\hline 10 & $\begin{array}{l}\text { Kamradt } \\
\text { M } \\
\text { Alemanha } \\
2017^{(24)}\end{array}$ & 404 & $\begin{array}{c}\text { Investigar o impacto de } \\
\text { características dos pacientes na } \\
\text { qualidade de vida relacionada à saúde de } \\
\text { pacientes multimórbidos com diabetes } \\
\text { mellitus tipo } 2 \text { em um ambiente } \\
\text { comunitário. }\end{array}$ & Observacional & EQ-5D \\
\hline & $\begin{array}{c}\text { Al- } \\
\text { Khaledi M }\end{array}$ & 503 & $\begin{array}{l}\text { Os objetivos deste estudo foram avaliar a } \\
\text { qualidade de vida relacionada à saúde }\end{array}$ & & SF-12 \\
\hline
\end{tabular}




\begin{tabular}{|c|c|c|c|c|c|}
\hline 11 & $\begin{array}{l}\text { Kuwait } \\
2018^{(17)}\end{array}$ & & $\begin{array}{l}\text { (QVRS) entre pacientes adultos com } \\
\text { diabetes atendidos em clínicas de diabetes } \\
\text { de cuidados primários de saúde no } \\
\text { Kuwait e examinar os fatores associados } \\
\text { à QVRS de pacientes com diabetes }\end{array}$ & Observacional & \\
\hline 12 & $\begin{array}{l}\text { Lima LR } \\
\text { Brasil } \\
2018^{(2)}\end{array}$ & 196 & $\begin{array}{l}\text { Avaliar a qualidade de vida dos } \\
\text { idosos com diabetes mellitus e } \\
\text { relacionar o tempo do diagnóstico do } \\
\text { diabetes com a qualidade de vida desses } \\
\text { idosos atendidos em uma unidade básica } \\
\text { de saúde. }\end{array}$ & Observacional & $\begin{array}{l}\text { WHOQOL- } \\
\text { BREF e } \\
\text { WHOQOL- } \\
\text { OLD }\end{array}$ \\
\hline 13 & $\begin{array}{l}\text { Alencar } \\
\text { DC } \\
\text { Brasil } \\
2019^{(22)}\end{array}$ & 50 & $\begin{array}{c}\text { Avaliar a qualidade de vida relacionada à } \\
\text { saúde de pessoas com diabetes mellitus } \\
\text { tipo } 2 \text { atendidas pela Atenção Primária à } \\
\text { Saúde }\end{array}$ & Observacional & SF-36 \\
\hline 14 & $\begin{array}{l}\text { Dhillon } \\
\text { Malásia } \\
2019^{(27)}\end{array}$ & 150 & $\begin{array}{l}\text { Investigar os três preditores possíveis a } \\
\text { seguir e sua relação com a QV entre } \\
\text { pacientes com DM2 na Malásia: } \\
\text { complicações e gravidade da doença, } \\
\text { adesão medicamentosa e bem-estar } \\
\text { psicossocial. }\end{array}$ & Observacional & AsianDQOL \\
\hline 15 & $\begin{array}{l}\text { Janssen J } \\
\text { Holanda } \\
2019^{(23)}\end{array}$ & 179 & $\begin{array}{l}\text { Avaliar as alterações nos sintomas } \\
\text { depressivos e na qualidade de vida } \\
\text { relacionada à saúde (QVRS) após a } \\
\text { triagem de comprometimento cognitivo } \\
\text { em pessoas com diabetes tipo } 2\end{array}$ & Observacional & $\begin{array}{c}\text { SF-36 e EQ- } \\
5 \mathrm{D}\end{array}$ \\
\hline 16 & $\begin{array}{l}\text { Paiva FTF } \\
\text { Brasil } \\
2019^{(20)}\end{array}$ & 196 & $\begin{array}{c}\text { Avaliar a dor e suas repercussões na } \\
\text { qualidade de vida de idosos com Diabetes } \\
\text { Mellitus. }\end{array}$ & Observacional & $\begin{array}{l}\text { WHOQOL- } \\
\text { BREF e } \\
\text { WHOQOL- } \\
\text { OLD }\end{array}$ \\
\hline
\end{tabular}

Fonte: elaborado pelos autores

A tabela 3 destaca os principais resultados e conclusões obtidos a partir da análise dos estudos, em que percebeu- se que o tema da qualidade de vida nos pacientes com diabetes acompanhados na Atenção
Primária relacionou- se principalmente a análise do perfil e a fatores que influenciam de forma positiva ou negativa na qualidade de vida desta população.

Quadro 3 - Descrição dos principais resultados e conclusões analisados nos estudos. Teresina, PI, Brasil, 2020.

\begin{tabular}{|l|l}
\hline $\mathbf{N}^{\mathbf{0}}$ & Resultados e conclusões \\
\hline
\end{tabular}




\begin{tabular}{|c|c|}
\hline 1 & $\begin{array}{l}\text { O diabetes exerce impacto significativo na vida dos idosos mais jovens (média de } 68,84 \\
\text { anos) do sexo feminino, com grau de escolaridade menor e menor tempo de diagnóstico } \\
\text { da doença. A maioria dos participantes manifestou um bom padrão de qualidade de vida } \\
\text { relacionada à saúde, e que o diabetes traz especificidades que variam de indivíduo para } \\
\text { indivíduo, caracterizando o fenômeno como singular. }\end{array}$ \\
\hline 2 & $\begin{array}{l}\text { Cerca de } 60 \% \text { dos pacientes do estudo apresentaram boa QVRS. As mulheres, } \\
\text { especialmente os malaios mais jovens, que tiveram diabetes por um período mais curto, } \\
\text { apresentaram melhor QVRS. No entanto, pacientes que não eram casados, apresentaram } \\
\text { dislipidemia e níveis mais altos de colesterol total e escores mais altos de PHQ-9 (escala } \\
\text { que mede sintomas depressivos) apresentaram menor QVRS. As complicações } \\
\text { macrovasculares apresentaram o maior efeito negativo sobre a QVRS. }\end{array}$ \\
\hline 3 & $\begin{array}{l}\text { No geral, o PEP resultou em uma melhoria significativa no SF-12 subescalas } \\
\text { emocionais, da dor e do papel e pontuações do utilitário SF-6D. Essas mudanças } \\
\text { positivas não foram associadas ao nível de participação. O PEP fez uma melhora } \\
\text { significativa na dor corporal, no papel emocional e nos aspectos gerais da QVRS. Maior } \\
\text { número de comparecimento à sessão não foi associado à melhoria da QVRS. }\end{array}$ \\
\hline 4 & $\begin{array}{l}\text { Este estudo encontrou uma associação entre discriminação percebida, comportamento } \\
\text { em saúde como dieta e componente mental da qualidade de vida. Embora não seja direta } \\
\text { foi encontrada associação com medidas biológicas quando ambas as raças foram } \\
\text { incluídas na análise, este estudo descobriu que a raça é um fator associado à } \\
\text { discriminação percebida e à influência da discriminação percebida pode diferir por raça. } \\
\text { Este estudo destaca a importância de adaptar culturalmente os programas de educação } \\
\text { em diabetes e levar em consideração fatores psicossociais, como discriminação } \\
\text { percebida ao trabalhar com os pacientes para melhorar o autocuidado, os resultados } \\
\text { clínicos e a qualidade de vida. }\end{array}$ \\
\hline 5 & $\begin{array}{l}\text { Este estudo mostrou que a comorbidade é altamente prevalente em pacientes com } \\
\text { diabetes tipo } 2 \text { e tem um impacto significativo sobre qualidade de vida física e mental, } \\
\text { em comparação com os sem comorbidades. A qualidade de vida física é reduzida } \\
\text { principalmente por distúrbios músculo- esqueléticos e cardiovasculares. Notou- se que a } \\
\text { qualidade de vida se deteriora significativamente com o aumento do número de } \\
\text { condições comórbidas. }\end{array}$ \\
\hline 6 & $\begin{array}{l}\text { O diabetes exerce um impacto significativo na vida dos idosos mais jovens do sexo } \\
\text { feminino, com escolaridade baixa e com menor tempo de diagnóstico da doença. Entre } \\
\text { os domínios estudados, a dor apresentou o pior escore, seguido dos aspectos sociais e do } \\
\text { estado geral de saúde. Os domínios com melhores escores foram: saúde mental, aspectos } \\
\text { emocionais, vitalidade, aspectos físicos e capacidade funcional. }\end{array}$ \\
\hline 7 & $\begin{array}{l}\text { A QV geral e a QVRS indicaram uma QV justa. Entre os domínios, o domínio físico foi } \\
\text { o pior com a menor pontuação média e as variáveis socioambientais eram relativamente } \\
\text { melhores. A QV geral foi fortemente associada a fatores físicos, psicológicos, sociais e } \\
\text { ambientais indicando uma má QV geral em todos os domínios. A QVRS foi } \\
\text { significativamente associada a fatores físicos, psicológicos e ambientais. A educação é } \\
\text { considerada independente preditor de boa QV em } 3 \text { dos } 4 \text { domínios do WHOQOL- } \\
\text { BREF. }\end{array}$ \\
\hline 8 & $\begin{array}{l}\text { A discriminação percebida foi significativamente associada ao estresse e serviu como } \\
\text { caminho para influenciar o componente de saúde mental da qualidade de vida (CSM). O } \\
\text { apoio social teve um efeito direto e indireto sobre o CSM através de uma associação } \\
\text { negativa com o estresse. Esses resultados sugerem que futuras intervenções devem ser }\end{array}$ \\
\hline
\end{tabular}




\begin{tabular}{|c|c|}
\hline & $\begin{array}{l}\text { desenvolvido para diminuir o estresse e aumentar o apoio social em torno da } \\
\text { discriminação para melhorar o CSM de qualidade de vida em pacientes com diabetes. }\end{array}$ \\
\hline 9 & $\begin{array}{l}\text { Na Atenção Primária à Saúde, aqueles que realizavam dietas e apresentavam } \\
\text { hemoglobina glicada } \leq 7 \% \text { apresentaram menor chance de pior qualidade de vida. As } \\
\text { mulheres apresentaram maiores chances de pior qualidade de vida que os homens. } \\
\text { Conclui-se neste estudo que, com exceção do tempo de diagnóstico e sexo, outras } \\
\text { variáveis que influenciaram a qualidade de vida dos diabéticos foram fatores } \\
\text { modificáveis (hemoglobina glicada, dieta alimentar e atividade física). }\end{array}$ \\
\hline 10 & $\begin{array}{l}\text { Destacou- se neste estudo que seis das } 14 \text { variáveis explicativas dos pacientes foram } \\
\text { significativamente associadas à pior QVRS, sendo elas: sexo feminino, escolaridade de } \\
\text { nove anos ou menos, restrições de mobilidade (físicas), presença de dor crônica, } \\
\text { angústia relacionada ao diabetes e índice de massa corporal. }\end{array}$ \\
\hline 11 & $\begin{array}{l}\text { A pontuação média geral da QVRS foi de } 61,7 \text { / 100. O estudo mostrou que os homens } \\
\text { apresentaram uma QV significativamente melhor que as mulheres e que o sexo feminino } \\
\text { e o relato de duas ou mais complicações diabéticas foram significativamente associados } \\
\text { a um composto de saúde física (APS) pobre. Pacientes com diabetes tipo } 2 \text { relataram } \\
\text { uma pontuação mediana da APS significativamente melhor em comparação com aqueles } \\
\text { com diabetes tipo } 1 \text {. }\end{array}$ \\
\hline 12 & $\begin{array}{l}\text { Os domínios do Whoqol-Bref com maiores escores, indicando melhor qualidade de vida } \\
\text { foram Relações Sociais e Psicológico, e uma pior qualidade de vida observada foi Meio } \\
\text { Ambiente. As facetas do Whoqol-Old com melhores escores foram: Intimidade e } \\
\text { Atividades passadas, presente e futura e a pior foi Morte e MorrerDestaca- se que os } \\
\text { idosos com mais de } 10 \text { anos diagnóstico apresentaram piores escores de qualidade de } \\
\text { vida no Físico, Relações Sociais, e nas facetas Autonomia, Participação Social, Morte e } \\
\text { Morrer. }\end{array}$ \\
\hline 13 & $\begin{array}{l}\text { Observou-se que em relação aos escores padronizados das dimensões do SF-36 } \\
\text { analisadas, os valores médios obtidos variaram entre } 51,26 \text { a } 72,10 \text {. Os maiores escores } \\
\text { médios foram verificados nos domínios de Capacidade Funcional, Vitalidade e Aspectos } \\
\text { Sociais, enquanto os menores escores médios foram obtidos nos domínios de Estado } \\
\text { Geral da Saúde. }\end{array}$ \\
\hline 14 & $\begin{array}{l}\text { Aproximadamente três quartos dos pacientes tiveram uma QV boa-excelente. As } \\
\text { variáveis relacionadas ao diabetes que foram significativamente associadas a escores } \\
\text { baixos de QV incluíram regimes de tratamento contendo insulina e controle glicêmico } \\
\text { deficiente. Os principais preditores de uma QV boa-excelente foram HbAlc } \leq 6,5 \% \text {, } \\
\text { níveis normais de ansiedade, adesão à medicação e escore Índice de Complicação e } \\
\text { Gravidade do Diabetes Adaptado (aDCSI) de um e dois em comparação com aqueles } \\
\text { maiores ou iguais a quatro. }\end{array}$ \\
\hline 15 & $\begin{array}{l}\mathrm{Na} \text { triagem, os participantes diagnosticados com comprometimento cognitivo } \\
\text { apresentaram significativamente mais sintomas depressivos e pior QVRS do que os nãos } \\
\text { suspeitos de comprometimento cognitivo durante a triagem (negativos na tela). }\end{array}$ \\
\hline 16 & $\begin{array}{l}\text { Neste estudo, os idosos que não referiram dor apresentaram melhores escores de QV do } \\
\text { que aqueles que queixaram- se de dor, exceto nos domínios físico e na faceta autonomia. } \\
\text { No grupo de idosos com DM que não referiram dor uma melhor QV foi observada no } \\
\text { domínio relações sociais e faceta intimidade e uma pior QV no domínio físico e faceta }\end{array}$ \\
\hline
\end{tabular}


autonomia.

Fonte: elaborado pelos autores

\section{DISCUSSÃO}

Esses resultados indicam que as pessoas com DM apresentam um bom padrão geral de QV (17-19,22,25,27). Resultados semelhantes foram encontrados em estudos observacionais realizados para investigar o impacto do DM na QV dos participantes, os quais demonstraram boa QV ${ }^{(22,28)}$. Acreditase que o entendimento de um bom estado de saúde esteja relacionado à ausência de complicações decorrentes do DM, que podem causar baixo impacto na $\mathrm{QV}$ dos pacientes (21).

Em relação às variáveis socioeconômicas, idosos mais jovens ${ }^{(18,25)}$ do sexo feminino (17,24) casados (18), com escolaridade igual ou inferior a nove anos (24) apresentaram piores escores de QV. Esses dados corroboram o estudo realizado para estimar a prevalência de DM determinada pela hemoglobina glicada na população brasileira, que constatou que a prevalência de diabetes é maior no sexo feminino, acima de 30 anos e com baixa escolaridade ${ }^{(29)}$. Esses dados demonstram que a população está adotando hábitos de vida pouco saudáveis no seu processo de envelhecimento.

Acredita-se que a piora da $\mathrm{QV}$ do idoso possa estar relacionada ao fato de que, ao receber o diagnóstico da doença, o indivíduo por não estar preparado para conviver com as limitações decorrentes do seu quadro clínico e mudanças no estilo de vida, manifesta sentimentos de angústia, insatisfação, decepção e fracasso ${ }^{(30)}$.

Em relação às variáveis clínicas, diabéticos com comorbidades e complicações expressam baixa qualidade de vida ${ }^{(15,18)}$. Pacientes com diabetes tipo 2 apresentam escore significativamente melhor ${ }^{(17)}$, menor tempo de diagnóstico ${ }^{(18)}$, boa adesão à medicação ${ }^{(26)}$ e controle glicêmico adequado $(26,27)$

Verifica-se que a duração da doença e o mau controle glicêmico favorecem o desenvolvimento de comorbidades que contribuem para o agravamento da saúde, o que requer outras terapias e cuidados e / ou tratamentos especializados ${ }^{(2,18)}$. No estudo de 1.676 pessoas com diabetes, as comorbidades que apresentaram redução significativa na QV física foram retinopatia, doença cardíaca, aterosclerose no abdômen ou nas pernas, doença pulmonar, incontinência, distúrbios nas costas, pescoço e ombros, osteoartrite e artrite reumatóide crônica ${ }^{(15)}$.

Quanto aos aspectos relacionados à QV, os domínios que indicaram os melhores padrões foram: Intimidade ${ }^{(2,20)}$, vitalidade $(21,22)$, atividades passadas, presentes e futuras 
(2), adesão à medicação (27), capacidade funcional ${ }^{(21,22)}$, dieta ${ }^{(26)}$ e bom controle da hemoglobina glicada $(\mathrm{HbA} 1 \mathrm{c} \leq 7 \%)^{(26,27)}$.

O controle glicêmico por meio da adesão à medicação foi estabelecido como um fator modificador de efeito nas relações entre HbA1c, depressão, ansiedade, gravidade da doença e $\mathrm{QV}^{(27)}$. Apesar de fortes evidências, a adesão terapêutica permanece baixa em pacientes com DM (31) sendo um fator preditivo que merece atenção no manejo desses pacientes.

Verifica-se também que, na APS, aqueles que faziam dieta e apresentavam hemoglobina glicada $\leq 7 \%$ apresentaram menor probabilidade de pior QV ${ }^{(26,27)}$, dados que corroboram estudo transversal realizado com 53 diabéticos, que evidenciou que níveis mais elevados de hemoglobina, colaboram para maior comprometimento da $\mathrm{QV}$ e maior percepção da gravidade da doença ${ }^{(32)}$.

Em contraste, os domínios dor ${ }^{(20,21,24)}$, meio ambiente ${ }^{(2,19)}$, saúde geral ${ }^{(21,22)}$, morte e morrer ${ }^{(2)}$, regimes de tratamento contendo insulina e controle glicêmico deficiente ${ }^{(27)}$, apresentaram piores escores gerais.

A dor está entre as principais causas limitantes na manutenção das atividades cotidianas do idoso, com implicações negativas na QV, bem como, em algumas circunstâncias, no estreitamento da vida social (33).

Ressalta-se também que aspectos físicos ${ }^{(19,21,22,24)}$, sociais ${ }^{(2,19-22)}$ e relacionados à saúde mental ${ }^{(2,19,21)}$ apresentaram escores https://doi.org/10.31011/reaid-2021-v.95-n.36-art.1169 Rev Enferm Atual In Derme v. 95, n. 36, 2021 e-021183 diferentes nos estudos selecionados, já demonstrando bons indicadores de $\mathrm{QV}$, por vezes indicando pior $\mathrm{QV}$.

Nesse ínterim, estudos indicam que as estratégias de educação em saúde e o empoderamento dos diabéticos favorecem a melhora da QV. Também destaca a necessidade de ajustes nos programas de educação em diabetes, a fim de levar em consideração fatores psicossociais e culturais para melhorar o autocuidado, os resultados clínicos e a QV ${ }^{(13,14)}$.

Dessa forma, são vistos os grupos de educação em diabetes promovidos pelas equipes de saúde da APS com o objetivo de estimular debates sobre questões relevantes para os indivíduos, sendo realizados por meio de rodas de conversa e temáticas definidas a partir das dúvidas dos participantes, a fim de fortalecer os laços de confiança com os profissionais de saúde e estimular a adesão às orientações fornecidas ${ }^{(34,35)}$.

Nesse contexto, fica evidente a necessidade de avaliar a qualidade de vida dos pacientes com DM no contexto da APS, pois saber o quanto o DM pode comprometer a QV do indivíduo permite identificar as necessidades de atenção e planejar os cuidados de enfermagem de forma individualizada e, assim, contribuir para a melhoria da QV e controle da doença ${ }^{(32)}$.

Esta revisão apresenta limitações, que estão relacionadas aos critérios de elegibilidade, em que apenas os artigos disponibilizados online gratuitamente, com a 
delimitação dos idiomas português, inglês e espanhol, dos últimos cinco anos, podem ter excluído estudos considerados importantes.

\section{CONCLUSÕES}

Os resultados deste estudo demonstram que as pessoas com Diabetes Mellitus acompanhadas na Atenção Básica apresentam bons padrões de qualidade de vida. Quanto aos aspectos relacionados à QV, verificou-se que os domínios que indicaram melhores padrões de QV foram: intimidade, vitalidade, atividades passadas, presentes e futuras, adesão medicamentosa, capacidade funcional, dieta alimentar e bom controle da hemoglobina glicada $(\mathrm{HbAlc} \leq 7 \%)$. Em contraste, os domínios dor, meio ambiente, saúde geral, morte e morrer, regimes de tratamento contendo insulina e controle glicêmico deficiente, apresentaram os piores escores gerais.

Porém, acredita-se que esta revisão permitiu o reconhecimento das dimensões que interferem na $\mathrm{QV}$ em pessoas com DM atendidas na APS, possibilitando o planejamento da implementação de ações pelas instâncias normativas, bem como pelos profissionais de saúde, direcionando suas práxis clínica, promover uma melhor qualidade de vida.

\section{REFERÊNCIAS}

1. World Health Organization. Definition, diagnosis and classification of diabetes mellitus and its complications. Geneva: WHO. 2009; 21 (2): 176-185. Disponível em: https://doi.org/10.1002/(SICI)1096-

9136(199807)15:7<539:AIDDIA668>3.0.CO;2-S.

2. Lima LRD, Funghetto SS, Volpe CRG, Santos WS, Funez MI, Stival MM. Quality of life and time of diagnosis of diabetes mellitus in the elderly. Brazilian Journal of Geriatrics and Gerontology [Internet]. 2018 [cited 2020 Apr 24]; 21 (2): 176-85. Available from: https://doi.org/10.1590/1981-

22562018021.170187.

3. International Diabetes Federation. Diabetes Atlas 2019 [acesso em 2020 abril 10]. Available http://www.diabetesatlas.org/

4. Cunha GHD, Barbosa RVA, Fontenele MSM, Lima MAC, Franco KB, Fechine FV. Insulin therapy waste produced in the households of people with diabetes monitored in Primary Care. Revista Brasileira de Enfermagem [Internet]. 2017 [cited $2020 \mathrm{Apr}$ 24]; 70(3): 618-25.Available from: https://doi.org/10.1590/0034-7167-20160406.

5. Vietta GG, Volpato G, Kretzer MR, da Gama FO, Nazário NO, Pereira E. (2019). Impacto do conhecimento nas atitudes, no sofrimento e qualidade de vida do paciente diabético. Arquivos Catarinenses de Medicina [Internet]. 2019 [cited 2020 Apr 24];, 48(4): 51-61. Available from: http://www.acm.org.br/acm/seer/index.php/ar quivos/article/view/520

6. Whoqol Group. The World Health Organization quality of life assessment (WHOQOL): position paper from the World Health Organization. Social science \& medicine [Internet]. 1995 [cited 2020 Apr 22]; 41(10): 1403-09. Available from: https://doi.org/10.1016/0277-9536(95)00112$\mathrm{K}$.

7. Maciel CL, Santos RM, Limborço Filho M, Assis IB, Marins FR. Impact of type 1 and 2 diabetes on the patient's quality of life. Revista Saúde em Foco [Internet]. 2018 
[acesso em 22 de abr 2020]; (10). Available from: http://portal.unisepe.com.br/unifia/wp content/uploads/sites/10001/2018/07/051_IM PACTO_DO_DIABETES_TIPO_1_E_2.pdf

8. Brasil. Ministério da Saúde. Portaria $n^{\circ}$. 2436 de 21 de setembro de 2017. Brasília: Diário oficial da República Federativa do Brasil, 2017. Disponível em: https://bvsms.saude.gov.br/bvs/saudelegis/gm /2017/prt2436_22_09_2017.html.

9. Ribeiro SP, Cavalcanti MDLT. Primary Health Care and Coordination of care: device to increase access and improve quality. Ciência \& Saúde Coletiva. 2020; 25: 17991808. Available from: https://www.scielo.br/j/csc/a/VJ9syfhhdCSqV HH4TbyxTJh/?lang=en

10. Whittemore R, Knafl K. The integrative review: updated methodology. J Adv Nurs [Internet]. 2005 [cited 2020 Apr 23]; 52(5):546-53.Available from: https://doi.org/10.1111/j.1365-

2648.2005.03621.x

11. Mendes KDS, Silveira RCDCP, Galvão CM. Integrative review: research method for incorporating evidence in health and nursing. Text \& context nursing. 2008; 17 (4), 758-64. Available from: https://www.scielo.br/j/tce/a/XzFkq6tjWs4w $\mathrm{HNqNjKJLkXQ} /$ ?lang=pt\&format=pdf

12. Moher D, Shamseer L, Clarke M, Ghersi D, Liberati A, Petticrew M, et al. Preferred reporting items for systematic review and meta-analysis protocols (PRISMA-P) 2015 statement. Syst Rev [Internet] 2015 [cited 2020 Apr 24];4:1. Available from: https://doi.org/10.1186/2046-4053-4-1.

13. Wong CK, Wong WC, Wan EY, Wong WH, Chan FW, Lam CL. Increased number of structured diabetes education attendance was not associated with the improvement in patient-reported health-related quality of life: results from Patient Empowerment Programme (PEP). Health Qual Life Outcomes [Internet]. 2015 [cited 2020 Apr 24];13:126. Available from: https://hqlo.biomedcentral.com/articles/10.11 86/s12955-015-0324-3.
14. Dawson AZ, Walker RJ, Campbell JA, \& Egede LE. Effect of perceived racial discrimination on self-care behaviors, glycemic control, and quality of life in adults with type 2 diabetes. Endocrine [Internet]. 2015 [cited 20 Apr 2020]; 49(2). Available from:

https://www.ncbi.nlm.nih.gov/pmc/articles/P MC4440842/.

15. Adriaanse MC, Drewes HW, van der Heide I, Struijs JN, Baan CA. The impact of comorbid chronic conditions on quality of life in type 2 diabetes patients. Qual Life Res [Internet]. 2016 [cited 22 Apr 2020]; 25(1). Available from: https://www.ncbi.nlm.nih.gov/pmc/articles/P MC4706581/.

16. Achuko O, Walker RJ, Campbell JA, Dawson AZ, Egede LE. Pathways Between Discrimination and Quality of Life in Patients with Type 2 Diabetes. Diabetes Technol Ther [Internet]. 2016 [cited 22 Apr 2020]; 18(3). Available from: https://www.ncbi.nlm.nih.gov/pmc/articles/P MC4790216/.

17. Al-Khaledi M, Al-Dousari H, Al-Dhufairi S, Al-Mousawi T, Al-Azemi R, Al-Azimi F, E. Badr H. Diabetes Self-Management: A Key to Better Health-Related Quality of Life in Patients with Diabetes. Med Princ Pract [Internet]. 2018 [cited 22 Apr 2020]; 27(4). Available from: https://doi.org/10.1159/000489310.

18. Chew BH, Sherina MS, Sazlina SG. Negative effects of diabetes-related distress on health-related quality of life: na evaluation among the adult patients with type 2 diabetes mellitus in three primary healthcare clinics in Malaysia. Health and Quality of Life Outcomes [Internet]. 2015 [cited 24 Apr 2020];13(1). Available from: https://www.ncbi.nlm.nih.gov/pmc/articles/P MC4657278/.

19. Sreedevi, A, Cherkil S, Kuttikattu DS, Kamalamma L, Oldenburg, B. Validation of WHOQOL-BREF in Malayalam and determinants of quality of life among people with type 2 diabetes in Kerala, India. Asia Pacific. Journal of Public Health [Internet]. 
2016 [cited 23 Apr 2020]; 28. Available from: https://doi.org/10.1177/1010539515605888.

20. Paiva FTF, Lima LR, Funez MI, Valpe CRG, Funguetto SS, Stival MM. The influence of pain in the quality of life of elderly people with Diabetes Mellitus. Rev Enfer UERJ [Internet]. 2019 [cited 22 Apr 2020];27. Available from: https://www.longdom.org/proceedings/theinfluence-of-pain-in-the-quality-of-life-ofelderly-with-diabetes-mellitus-44340.html.

21. Sousa EL, Martins MM, Costa MS, Moreira MRC, Silva AO. Quality of life and factors associated with the health of elderly diabetics. Rev Enferm UERJ [Internet]. 2016 [cited 23 Apr 2020]; 24 (5). Available from: https://doi.org/10.12957/reuerj.2016.8456.

22. Alencar DC, Lages Filho PF, Neiva MJLM, Alencar AMPG, Moreira WC, Ibiapina ARS. Dimensions of the quality of life negatively affected in people living with Diabetes Mellitus. J. res. fundam. Care Online [Internet]. 2019 [cited 23 Apr 2020]; 11 (1). Available from: https://doi.org/10.9789/21755361.2019.v11i1.199-204.

23. Janssen J, Koekkoek PS, Biessels G, Kappelle JL, Rutten GEHM, On behalf of the Cog-ID study group. Depressive symptoms and quality of life after screening for cognitive impairment in patients with type 2 diabetes: observations from the Cog-ID cohort study. BMJ Open[Internet]. 2019 [cited 23 Apr 2020]; 9. Available from: https://bmjopen.bmj.com/content/9/1/e024696

24. Kamradt M, Krisam J, Kiel M, Qreini M, Besier W, Szecsenyi J, Ose D. Health-Related Quality of Life in Primary Care: Which Aspects Matter in Multimorbid Patients with Type 2 Diabetes Mellitus in a Community Setting? Plos one [Internet]. 2017 [cited 22 Apr 2020]; 12(1). Available from: https://doi.org/10.1371/journal.pone.0170883.

25. Leite ES, Lubenow JAM, Moreira MRC, Martins MM, Costa IP, Silva AO. Evaluation of the Impact of Diabetes Mellitus on the quality of life of the elderly. Cienc Cuid Saude. 2015; 14 (1). Available from:
https://periodicos.uem.br/ojs/index.php/Cienc CuidSaude/article/view/21353

26. Corrêa K, Gouvêa GR, Silva MAV, Possobon RF, Barbosa LFLN, Pereira AC, Miranda LG, Cortellazzi KL. Quality of life and characteristics of diabetic patients. Ciênc Saúde Pública [Internet]. 2017 [cited 23 Apr 2020];22(3). Available from: https://doi.org/10.1590/1413-

81232017223.24452015 .

27. Dhillon $\mathrm{H}$, Nordin RB, Ramadas A. Quality of Life and Associated Factors among Primary Care Asian Patients with Type 2 Diabetes Mellitus. Int J Environ Res Public Health [Internet]. 2019 [cited 23 Apr 2020]; 16(19). Available from: https://doi.org/10.3390/ijerph16193561.

28. Braga NS, Silveira VFSB, Gonçalves NEXM. Impact of diabetes mellitus on patients' quality of life: a survey through social networks. Science and Praxis. 2019;12 (23).

Available

from: https://revista.uemg.br/index.php/praxys/articl e/view/4091

29. Malta DC, Duncan BB, Schmidt MI, Machado ÍE, Silva AGD, Bernal RTI, Pereira CA, Damacena GN, Stopa SR, Rosenfeld LG, Szwarcwald CL. Prevalence of diabetes mellitus determined by glycated hemoglobin in the Brazilian adult population, National Health Survey. Revista Brasileira de Epidemiologia [Internet]. 2019 [cited 24 Apr 2020]; 22. Available from: https://doi.org/10.1590/1980549720190006.supl.2

30. Marques JS, Brandão SADSM, Lima ACG, de Oliveira AC, Oliveira MM, Gomes AT, Leal, SRMD. Qualidade de vida de diabéticos acompanhados pela Estratégia Saúde da Família. Revista Cubana de Enfermería [Internet]. 20212015 [cited 20 Apr 2020];37(1). Available from:http://www.revenfermeria.sld.cu/index.p hp/enf/article/view/3536

31. Baldoni NR, Oliveira REM, Franco LJ, Fabbro ALD. Adherence to the pharmacological treatment of people with type 2 Diabetes Mellitus. Rev. Bras. Farm. Hosp. Serv. Health [Internet]. 2016 [cited 24 
Apr 2020]; 7 (4). Available from: https://doi.org/10.1016/j.amjmed.2005.04.012

32. Tonetto IFA, Baptista MHB, Gomides DS, Pace AE. Quality of life of people with diabetes mellitus. Rev Esc Enferm USP [Internet]. 2019 [cited 24 Apr 2020]; 53 (e03424). Available from: https://doi.org/10.1590/S1980220X2018002803424.

33. Meneses MO, Marques JS, Gomes AT, Brandão SADSM, Leal, SRMD, Oliveira VAS (2021). Conhecimento e atitudes de pacientes frente a medidas preventivas do pé diabético. Revista Enfermagem Atual In Derme [Internet]. 2021 [cited 23 Apr 2020]; 95(34). Available from: https://www.revistaenfermagematual.com/ind ex.php/revista/article/view/1034

34. Salci MA, Meirelles BHS, Silva DMGV. Primary care for diabetes mellitus patients from the perspective of the care model for chronic conditions. Rev. Latino-Am. Nursing [Internet]. 2017 [cited 22 Apr 2020]; 25.
Available from: https://doi.org/10.1590/15188345.1474.2882.

35. Moreschi C, Rempel C, Backes DS, Pombo CNF, Siqueira DF, Pissaia LF. Actions of FHS teams for the quality of life of people with diabetes 1. Cienc Cuid Saude [Internet]. 2018 [cited 22 Apr 2020];17(2). Available from: https://doi.org/10.4025/cienccuidsaude.v17i2. 41000.

\section{Autor correspondente}

Jaciane Santos Marques, Campus Universitário Ministro Petrônio Portella, Ininga, Teresina - PI, 64049-550, +55 (86) 3215-5513, E-mail: jacianesantosmarques@hotmail.com

Submissão: 2021-07-06

Aprovado: 2021-12-22 